\title{
Automatic expert system based on images for accuracy crop row detection in maize fields
}

\author{
J.M. Guerrero ，M. Guijarro , M. Montalvo , J. Romeo , L. Emmi , A. Ribeiro , G. Pajares
}

\begin{abstract}
A B S T R A C T
This paper proposes an automatic expert system for accuracy crop row detection in maize fields based on images acquired from a vision system. Different applications in maize, particularly those based on site specific treatments, require the identification of the crop rows. The vision system is designed with a defined geometry and installed onboard a mobile agricultural vehicle, i.e. submitted to vibrations, gyros or uncontrolled movements. Crop rows can be estimated by applying geometrical parameters under image perspective projection. Because of the above undesired effects, most often, the estimation results inaccurate as compared to the real crop rows. The proposed expert system exploits the human knowledge which is mapped into two modules based on image processing techniques. The first one is intended for separating green plants (crops and weeds) from the rest (soil, stones and others). The second one is based on the system geometry where the expected crop lines are mapped onto the image and then a correction is applied through the well-tested and robust Theil-Sen estimator in order to adjust them to the real ones. Its performance is favorably compared against the classical Pearson product-moment correlation coefficient.
\end{abstract}

\section{Introduction}

\subsection{Problem statement}

Machine vision systems onboard robots are being increasingly used for site specific treatments in agriculture. With such arrangements, the robot navigates and acts over a site-specific area of a larger farm (Davies, Casady, \& Massey, 1998), where the vision systems can supply abundant information.

An important issue related with the application of machine vision methods is that concerning the crop row and weed detection, which has attracted numerous studies in this area (Burgos-Artizzu, Ribeiro, Tellaeche, Pajares, \& Fernández-Quintanilla, 2009; Guerrero, Pajares, Montalvo, Romeo, \& Guijarro, 2012; LópezGranados, 2011; Montalvo et al., 2012; Onyango \& Marchant, 2003; Sainz-Costa, Ribeiro, Burgos-Artizzu, Guijarro, \& Pajares, 2011; Tellaeche, Burgos-Artizzu, Pajares, \& Ribeiro, 2008; Tellaeche, Burgos-Artizzu, Pajares, Ribeiro, \& Fernández-Quintanilla, 2008). The goal is to eliminate weeds to favor the growth of crops.

The vision system consists of a CCD-based calibrated camera with known intrinsic parameters, i.e. focal length, lens distortion, image center and CCD sensor sizes and pixel resolutions. The camera is located in front of the robot, inclined with a tilt angle (pitch) and at a high from the ground. Yaw and roll angles are also known. This allows determining the rotation and translation matrices defining the extrinsic parameters. Thus, areas in the field can be identified onto the image plane. This means that given an element in the field, with its spatial location, we can determine its relative positioning on the image.

The vehicle navigates on a real terrain presenting irregularities and roughness. This produces vibrations and also swinging mainly in the pitch and roll angles. The yaw angle is assumed to be correct because otherwise the robot navigates erroneously out of the crop rows. Moreover, the spacing of crop rows in the field is also known. Because of the above, most often, the mapped expected crop rows in the image do not match with the real ones, this inaccurate estimation impedes the application of correct site specific treatments. On the other hand the discrimination of crops and weeds in the image is a very difficult task because their Red, Green and Blue spectral components display similar values. This means that no discrimination is possible between crops and weeds based on the spectral signatures. Thus, the best option is to locate the crop rows in the image with the maximum accuracy as possible. Indeed, if the crop rows are well located, we can accurately identify those pixels along and around the detected line as crops and the remainder, which are moved away, can be considered as weeds. To achieve 
this goal, we propose an automatic expert system, which exploits the human knowledge, with two main modules based on image processing techniques, as described later.

\subsection{Revision of methods}

Several strategies have been proposed for crop row detection. Fontaine and Crowe (2006) tested the abilities of fourth line detection algorithms to determine the position and the angle of the camera with respect to a set of artificial rows with and without simulated weeds. These were stripe analysis, Hough transform, blob analysis and linear regression. The following is a list of crop row detection methods grouped into different categories including the above.

\subsubsection{Methods based on the exploration of horizontal strips}

Søgaard and Olsen (2003) apply RGB color image transformation to gray scale. This is done by first dividing the color image into its red, green and blue channels and then by applying the well-tested methods to extract living plant tissue described in Woebbecke, Meyer, von Bargen, and Mortensen (1995). After this, the gray scale image is divided into horizontal strips where maximum gray values indicate the presence of a candidate row, each maximum determines a row segment and the center of gravity of the segment is marked at this strip position. Crop rows are identified by joining marked points through a similar method to the one utilized in the Hough transform or by applying linear regression. Sainz-Costa et al. (2011) have developed a strategy based on analysis of video sequences for identifying crop rows. Crop rows persist along the directions defined by the perspective projection with respect the 3D scene in the field. Exploiting this fact, they apply gray scale transformation based on the approach proposed by Ribeiro, Fernández-Quintanilla, Barroso, and García-Alegre (2005) and then the image is binarized applying a thresholding technique. Each image is divided into four horizontal strips. Rectangular patches are drawn over the binary image to identify patches of crops and rows. The gravity centers of these patches are used as the points defining the crop rows and a line is adjusted considering these points. The first frame in the sequence is used as a lookup table that guides the full process for determining positions where the next patches in subsequent frames are to be identified. Hague, Tillet, and Wheeler (2006) transform the original RGB image to gray scale. The transformed image is then divided into eight horizontal bands. The intensity of the pixels across these bands exhibits a periodic variation, due to the parallel crop rows. Since the camera characteristics, pose and the crop row spacing are known a priori, the row spacing in image pixels can be calculated for each of the horizontal bands using a pinhole model of the camera optics. A band-pass filter can then be constructed which will enhance this pattern, and has a given frequency domain response. Sometimes horizontal patterns are difficult to extract because crops and weeds form a unique patch.

\subsubsection{Methods based on the Hough transformation}

According to Slaughter, Giles, and Downey (2008), one of the most commonly used machine vision methods for identifying crop rows is based upon the Hough (1962) transform. It was intended to deal with discontinuous lines, where the crop stand is incomplete with gaps in crop rows due to poor germination or other factors that result in missing crop plants in the row. It has been intended for realtime automatic guidance of agricultural vehicles (Astrand \& Baerveldt, 2005; Hague, Marchant, \& Tillett, 1997; Leemans \& Destain, 2006; Marchant, 1996). It is applied to binary images, which are obtained by applying similar techniques to the ones explained above, i.e. RGB image transformation to gray scale and binarization (Tellaeche, Pajares, Burgos-Artizzu, \& Ribeiro, 2011; Tellaeche et al.,
2008; Tellaeche, Burgos-Artizzu, Pajares, Ribeiro, et al., 2008). Gée, Bossu, Jones, and Truchetet (2008) apply a double Hough transform under the assumption that crop rows are the only lines of the image converging to the vanishing point, the remainder lines are rejected, additional constraints such as inter-row spacing and perspective geometry concepts help to identify the lines. It is required to determine the threshold required by the Hough transform to determine maximum peaks values (Jones, Gée, \& Truchetet, 2009a, 2009b) or predominant peaks (Rovira-Más, Zhang, Reid, \& Will, 2005). Depending on the crop densities several lines could be feasible and a posterior merging process is applied to lines with similar parameters (Tellaeche et al., 2008; Tellaeche, Burgos-Artizzu, Pajares, Ribeiro, et al., 2008; Tellaeche et al., 2011). Although intended for real-time, as mentioned before, in our images, where crop and weed plants contribute on the Hough parameter estimation, this method becomes computationally expensive (Ji, \& Qi, 2011). On the other hand, the randomized Hough transform requires selecting pairs of points to be considered as a line, i.e. pairs of points belonging to a crop row. If we apply this technique in images where edge points have been extracted, the selection of those pairs becomes highly complex because weeds are also involved.

\subsubsection{Vanishing point-based}

Pla, Sanchiz, Marchant, and Brivot (1997) propose an approach that identifies regions (crops/weeds and soil) by applying color image segmentation. They use the skeleton of each defined region as a feature to work out the lines that define the crop. The resulting skeletons and their properties, defined as chains of connected contour points, allow the identification of crop rows oriented toward the vanishing point. This process is highly dependent of skeletons, which are not always easy to extract, specially taking into account that weed patches are present. Romeo et al. (2012) apply also knowledge concerning the position of the vanishing point and the crop rows arrangement in the field to detect the expected crop rows. The process is based on the identification of maximum accumulation of green pixels along lines oriented toward the vanishing point. A supervised fuzzy clustering method is the proposed strategy for greenness identification. This makes the method highly dependent on the training phase unlike the one prosed in this approach which is automatic, i.e. unsupervised.

\subsubsection{Stereo-based approach}

Kise, Zhang, and Rovira-Más (2005) or Kise and Zhang (2008) developed a stereovision-based agricultural machinery crop-row tracking navigation system. Stereo-image processing is used to determine 3D locations of the scene points of the objects of interest from the obtained stereo image. Those 3D positions, determined by means of stereo image disparity computation, provide the base information to create an elevation map that uses a 2D array with varying intensity to indicate the height of the crop. This approach requires crops with significant heights with respect the ground. Because in maize fields, during the treatment stage, the heights are not relevant, it becomes ineffective in our application. Rovira-Más, Zhang, and Reid (2008) have applied and extended stereovision techniques to other areas inside Precision Agriculture. Only feasible if crops or weeds in the 3D scene display a relevant height.

\subsubsection{Methods based on blob analysis}

This method finds and characterizes regions of contiguous pixels of the same value in a binarized image (Fontaine \& Crowe, 2006). The algorithm searches for white blobs (inter-row spaces) of more than 200 pixels, under the assumption that smaller blobs could represent noise in the crop rows. Once the blobs were identified, the algorithm determined the angle of their principal axes and the location of their centre of gravity. For a perfectly straight white stripe, the centre of gravity of the blob was over the centre 
line of the white stripe, and the angle was representative of the angle of the inter-row spaces. The algorithm returned the angle and center of gravity of the blob closest to the center of the image. Identification of blobs in areas with weed patches does not distinguish between blobs caused by weeds and crops.

\subsubsection{Methods based on the accumulation of green plants}

Olsen (1995) proposed a method based on the consideration that along the crop row appear an important accumulation of green parts in the image. The image is gray scale transformed where green parts appear clearer that the rest. A sum-curve of gray levels is obtained for a given rectangular region exploring all columns in the rectangle. It is assumed that vertical lines follow this direction in the image. The images are free of perspective projection because they are acquired with the camera in orthogonal position. A sinusoidal curve is fitted by means of least squares to the sum-curve previously obtained. Local maxima of the sinusoid provide row centers locations.

\subsubsection{Methods based on frequency analysis}

Because crop rows are vertical in the 3D scene, they are mapped under perspective projection onto the image displaying some behavior in frequency domain. Vioix et al. (2002) exploit this feature and apply a bi-dimensional Gabor filter, defined as a modulation of a Gaussian function by a cosine signal. The frequency parameter required by the Gabor filter is empirically deduced from the 2D-Fast Fourier Transform (Bossu, Gée, Guillemin, \& Truchetet, 2006). Bossu, Gée, Jones, and Truchetet (2009) apply wavelets to discriminate crop rows based on the frequency analysis. They exploit the fact that crop rows are well localized in the frequency domain; thus selecting a mother wavelet function with this frequency the crop rows can be extracted. Crops, in the images we have studied, do not display clear frequency contents in the Fourier space, therefore the application of filters based on the frequency becomes a difficult task.

\subsubsection{Methods based on linear regression}

Some techniques above apply this approach. Billingsley and Schoenfisch (1997) reported a crop detection system that is relatively insensitive to additional visual 'noise' from weeds. They used linear regression in each of three crop row segments considered and a cost function analogous to the moment of the best-fit line to detect lines fitted to outliers (i.e., noise and weeds) as a means of identifying row guidance information. Montalvo et al. (2012) apply a linear regression for crop row detection in images containing high weeds densities. Some templates are used to guide the detection. Linear regression is also applied in Søgaard and Olsen (2003). Linear regression is highly sensitive to isolated weeds patches placed on the inter crop rows and also for weeds patches overlapped with crops. In this paper we also apply linear regression based on the Theil Sen estimator (Sen, 1968; Theil, 1950) which is free of the above sensitivity and has been proven in statistics with satisfactory results.

\section{Design of the automatic expert system}

\subsection{System architecture}

The system architecture is inspired on the human expert knowledge about the specific application and also considering the requirements that must be fulfilled. Astrand (2008) and Slaughter et al. (2008) propose a list of requirements for guidance systems that can be also considered for crop row detection, which in essence is a similar problem. Knowledge and requirements are mapped as follows to build the architecture of the proposed automatic expert system for the accuracy crop row detection based on images.

(a) Both crop and weeds display similar color spectral components and during the treatment their growth stages are similar, i.e. with similar height in the plants.

(b) Crop rows are accumulations of green plants following specific alignments oriented to the vanishing point. Crops are sown, not manually planted, and the inter-line distances in the field are known.

(c) Weeds appear on isolated or overlapped patches with respect crops with irregular distributions.

(d) Crop rows must be located with the most accuracy as possible, regardless the distribution of weeds patches around crop and also considering that crop plants could miss along crop lines, as a common situation.

\section{Expert system Crop row detection}

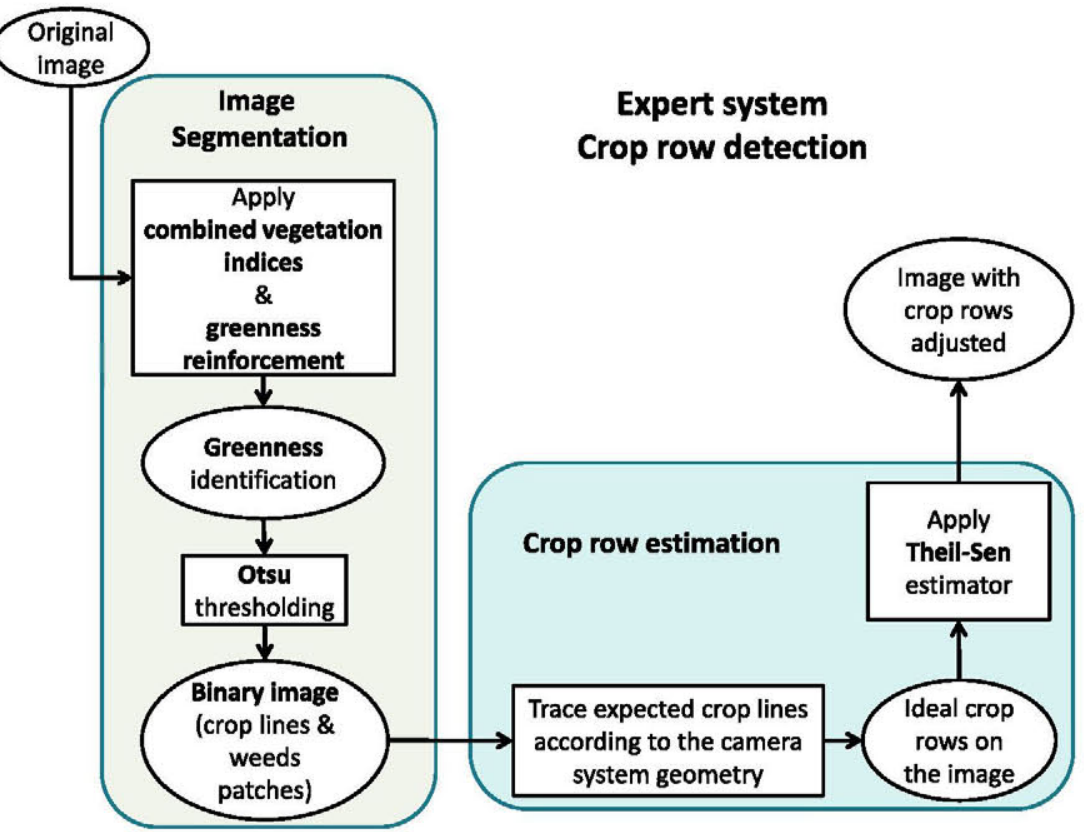

Fig. 1. Automatic expert system architecture. 
(e) Camera system geometry is known, i.e. the intrinsic and extrinsic parameters.

(f) The robot navigates on uneven terrains with perhaps abundant irregularities.

(g) The system must work on real-time. This represents a tradeoff between the speed of the robot and the computational cost.

Based on this knowledge and requirements and also considering advantages and shortcomings of the different crop row detection methods, the automatic expert system is designed consisting of two main modules: image segmentation and crop rows estimation. Fig. 1 displays schematically these two modules with the corresponding processes. This results in a robust expert system, making the contribution of this paper.

\subsection{Image segmentation}

Image segmentation is focused on the separation of green plants (crops and weeds) from the rest (soil, stones and others). According to point (a) in the list of knowledge and requirements above, the best option to identify weeds and crop is the application of vegetation indices instead of methods based on height discrimination. Vegetation indices are well tested methods, Guijarro et al. (2011) propose a combination of vegetation indices, which is the one chosen in this paper because its performance in maize fields.

\subsubsection{Combination of vegetation indices}

Given an original input image in the RGB color space, we apply the following normalization scheme, which is usually applied in agronomic image segmentation (Gée et al., 2008),

$r=\frac{R_{n}}{R_{n}+G_{n}+B_{n}}, \quad g=\frac{G_{n}}{R_{n}+G_{n}+B_{n}}, \quad b=\frac{B_{n}}{R_{n}+G_{n}+B_{n}}$

where $R, G$ and $B$ are the normalized RGB coordinates ranging from 0 to 1 and are obtained as follows:

$R_{n}=\frac{R}{R_{\max }}, \quad G_{n}=\frac{G}{G_{\max }}, \quad B_{n}=\frac{B}{B_{\max }}$

where $R_{\max }=G_{\max }=B_{\max }=255$ for our 24-bit color images.

Vegetation indices to be combined are computed as follows Guijarro et al. (2011),

Excess Green (Woebbecke et al., 1995; Ribeiro et al., 2005)
Color index of vegetation extraction (Kataoka, Kaneko,$$
\text { CIVE }=0.441 r-0.811 \mathrm{~g}
$$$$
+0.385 b+18.78745
$$
Okamoto, \& Hata, 2003)

$$
E x G=2 g-r-b
$$

Vegetativen

(Hague et al., 2006)

$$
V E G=\frac{g}{r^{a} b^{1-a}},
$$

with $a=0.667$ as in its reference

Excess green minus excess red (Meyer \& Camargo-Neto, 2008; Neto, 2004)

$$
E x G R=E x G-E x R
$$

where excess red is computed as follows (Meyer, Hindman, \& Lakshmi 1998): ExR $=1.4 r-g$. According to Guijarro et al. (2011) the above four indices are combined to obtain the resulting value COM as follows,

$C O M=w_{E x G} E x G+w_{E X G} E x G R+w_{C I V E} C I V E+w_{V E G} V E G$ where $w_{E X G}=0.25, w_{E X G R}=0.30, w_{C I V E}=0.33$ and $w_{V E G}=0.12$ are the weights for each index, representing their relative relevance in the combination. The resulting combined image COM, is linearly mapped to range into the interval $[0,1]$.

\subsubsection{Greenness reinforcement}

Romeo et al. (2012) propose a fuzzy clustering strategy where the cluster containing pixels belonging to green plants has been analyzed. Clusters contain pixels with the three spectral components in the RGB model as features. Obviously, and as expected, the green spectral component is dominant. On average this component in the cluster center for green plants represents values above the $36 \%$ with respect the other two components. Exploiting this knowledge and applying the trivial reasoning that pixels coming from plants should have their green component dominant, we accentuate the greenness in COM by multiplying their values by $g$ in Eq. (1), i.e. a new greenness is obtained as: $G A=C^{2} M^{*} g$. The multiplication is carried out pixel by pixel and GA is linearly mapped to range in $[0,1]$. Because $g$ represents the percentage of the green component, the result obtained represents the emphasis in the greenness.

\subsubsection{Thresholding}

Given the transformed image GA, the next step is its binarization for posterior processing. An easy threshold based on the mean gray level of the image (histogram) has been implemented in Gée et al. (2008) where the living plant material (crop or weed) appears as white spots and the rest (i.e. soil surface, stones, shadows) as black. Also in Guijarro et al. (2011) the well-known Otsu's (1979) method, traditionally applied for binarization, has been applied. More complex approaches have been also applied such as the one used in Bossu et al. (2009), based on the $k$-means clustering method. We have chosen the Otsu's method for its well-known performance as reported in Meyer and Camargo-Neto (2008) and also based on the study of Sezgin and Sankur (2004) where its performance has been tested in images where the number of pixels in both parts of the image histogram that Otsu's produces is close to each other.

Fig. 2(a) displays an original image in the RGB color space of a maize crop field. The color space transformation by applying GA is displayed in Fig. 2(b). Fig. 2(c) displays the image transformation from image in Fig. 2(b) by applying the Otsu's method. Note the landmarks in the image, which are explained later in Section 3.

\subsection{Crop row estimation}

This module is intended to apply the knowledge embedded in points (b)-(f), Section 2.1, at the same time it provides specific solutions for the requirements expressed in such points.

\subsubsection{Tracing expected crop lines}

The robot navigates on uneven terrains with perhaps abundant irregularities, the knowledge of extrinsic parameters of the vision system does not suffice because the camera is continuously involved in a permanent swinging. We propose the customization of the Theil-Sen regression estimation approach, because of its well-tested performance in statistics.

Because the crop rows arrangement are known in the field and also the extrinsic and intrinsic camera system parameters, the expected crop row locations in the image can be estimated and mapped as known lines onto the image (Fu, González, \& Lee, 1987; Hartley \& Zisserman, 2006). Under the assumption of ideal system geometry the expected lines should match and overlap the imaged real crop rows. Nevertheless, due to uneven terrains and errors in the crop row alignment during the sowing, this often does not occur. 


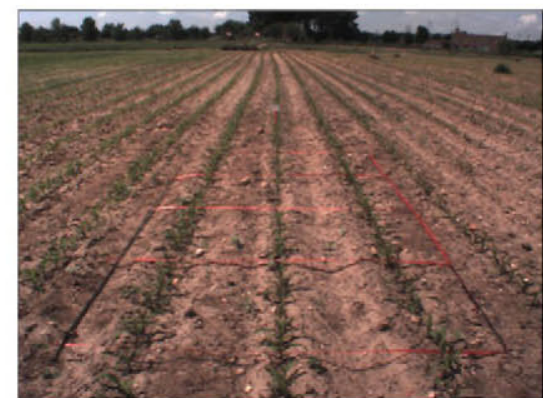

(a)

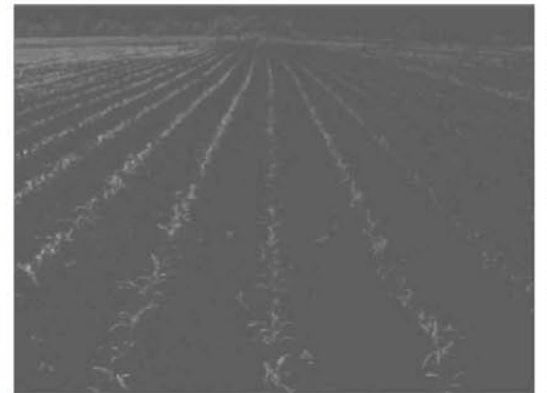

(b)

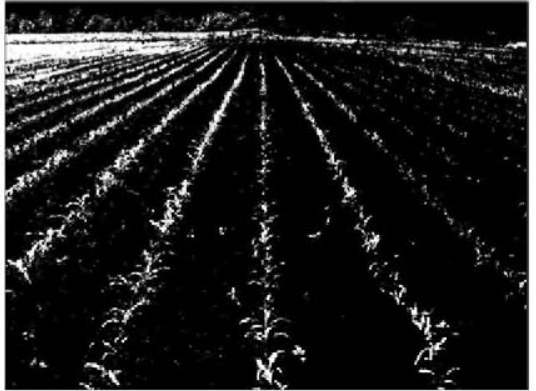

(c)

Fig. 2. (a) Original image; (b) GA index extracted from the image in (a); (c) binary image after Otsu thresholding.

Therefore, under the above consideration, two cases can appear with respect to the expected and the imaged real crop lines: (a) they match; (b) they do not match. In the first case, the detection method needs to verify this matching. In the second case, a line location correction must be applied until the real crop row is located. Under this approach the system geometry through the intrinsic and extrinsic parameters guides the crop row detection process.

Now the question is: how can we verify the expected lines match or not with the real crop ones? Because we have available white pixels representing green plants in the binary image, we can adjust a straight line for specific pixel alignments that are expected to identify crop rows. This will represent the real crop line. So, because we have both, the expected straight line equation and the adjusted one, we are able to verify the correct or incorrect match for both lines. Thus, we focus the effort in methods for estimating the parameters defining real crop lines.

\subsubsection{Correction of the expected crop lines: Theil-Sen estimator}

An important problem to be addressed in our approach is that the method selected can cope with specific pixel alignments but also must be robust enough to avoid significant deviations caused by weeds that are not aligned and placed more or less near the main crop row alignments. This is the main issue addressed in this work.

Stewart (1999) provides a tutorial oriented toward robust parameter estimation in computer vision. Two frequently techniques used are least-median of squares (LMS) (Rousseeuw, 1984) and M-estimators (Hampel, Rousseeuw, Ronchetti, \& Stahel, 1986; Huber, 1981), but a huge volume of data implies that parameter estimation techniques in computer vision are heavily over constrained, even for problems where low-level feature extraction, such as edge detection, are applied. This in turn implies that parameter estimation problems in vision should be solved by least squares or, more generally, maximum likelihood estimation (MLE) techniques. Unfortunately, computer vision data are rarely drawn from a single statistical population as required for effective use of MLE.

In our approach we must estimate two parameters, defining the straight line equations associated to the corresponding crop rows, they are the slope $\alpha$ and the intercept $\beta$. For the linear regression approach, after several studies, we observed that the least squares estimator of a regression, coefficient $\alpha$ is vulnerable to gross errors and the associated confidence interval is, in addition, sensitive to non-normality of the parent distribution. With other measures, for example, the breakdown point (Rousseeuw \& Leroy, 1987) a small number of outlying data can cause an estimate to diverge arbitrarily far from the true estimate. From the point of view of our approach, this means that few weed pixels can move the least squares fit far from the true fit, i.e. far of the real crop line. A second measure of robustness is the influence function (Hampel et al., 1986; Huber, 1981), in which the change in an estimate caused by insertion of outlying data, using a function of the distance, also causes false estimations because it should tend to zero with increasing distance to achieve robustness.

Alternative estimators for the regression coefficient, $\alpha$, based on suitable rank tests are proposed by Mood (1950). They apply the estimation of both parameters $\alpha$ and $\beta$ simultaneously using the statistical median by trial and error. Adichie (1967) proposes a more restrictive method under the assumption that the set of points to be adjusted is an absolutely continuous and symmetric distribution function with also an absolutely continuous and square integrable density function; Theil (1950) proposes a very simple estimator for $\alpha$ using also the statistical median; Dytham (2011) and Sen (1968) study a simple and robust estimator for $\alpha$ based on Kendall's (1955) tau rank correlation, a simple nonparametric test that can be used instead of normal regression. Hence, the estimator for $\alpha$ and $\beta$ is the median of the set of slopes, where a simple slope is computed between every possible pair of pixels $i$ and $j$ with image coordinates $\left(x_{i}, y_{i}\right)$ and $\left(x_{j}, y_{j}\right)$ respectively, finally the median slope is then selected as the best estimate for $\alpha$.

Based on the above considerations, we select the Theil-Sen estimator as proposed in Massart (1997), because of its statistical efficiency and its robustness, even for low image resolutions, resulting in a promising approach in agricultural images containing crop rows. Nevertheless, as we will see later, its effectiveness from the real-time point of view is relatively low. This means that further analysis or new software and hardware implementations should be required for real-time processing.

A straight line is represent by its slope $\alpha$ and its intercept $\beta$ as follows,

$Y=\alpha X+\beta$

Given a distribution of $n$ pixels the goal is to adjust a straight line to such distribution. The Theil-Sen estimator evaluates pairs of pixels $i$ and $j$ and compute the slope over the set of all possible pairs of such pixels, i.e. over the $n(n-1) / 2$ possible combinations. This is carried out as follows,

$\alpha=\operatorname{med}\left(S_{i j} \mid S_{i j}=\left(\frac{y_{j}-y_{i}}{x_{j}-x_{i}+\varepsilon}\right)\right) ; \quad x_{i} \neq x_{j}, i, j=1,2, \ldots, n$

The estimation of the intercept, $\beta$, is computed as the statistical median of the intercepts obtained with the robust slope $\alpha$ in (9). The $\varepsilon$ parameter, set to $10^{-3}$, is introduced to avoid exactly vertical lines with slope toward $\infty$. Polar coordinates could be used to avoid this problem. Nevertheless, vertical lines do not appear in our real application. This is carried out as follows,

$\beta=\operatorname{med}\left(y_{i}-\alpha x_{i}\right) ; \quad \forall i=1,2, \ldots, n$ 


\section{Results}

The images used for this study belong to maize crops. They were captured with a BASLER 17FC 1400 color camera during April/May 2011 in a 1.7-ha experimental field of maize on La Poveda Research Station, Arganda del Rey, Madrid. All acquisitions were spaced by five/six days, i.e. they were obtained under different environmental lighting conditions and different growth stages in maize and weed plants. The digital images were captured under perspective projection and stored as 24-bit color images with resolutions of $1392 \times 1038$ pixels saved in RGB (Red, Green and Blue) color space in the TIFF format. The images were processed under LabVIEW Real-Time (2012) from National Instruments, release 2011, under a CompactRIO-9082 $1.33 \mathrm{GHz}$ dual-core Intel Core i7 processor, including LX150 FPGA with Real-Time Operating System. The proposed algorithm is developed in $\mathrm{C++}$ with MS Visual Studio and compiled as a DLL, which is embedded as an additional module in LabVIEW. A set of 240 images was processed. This equipment is intended to fulfill the real-time specifications expressed in point $(\mathrm{g})$ Section 2.1 .

The camera extrinsic and intrinsic parameters are: pitch angle $=20^{\circ}$, roll angle $=0^{\circ}$ and yaw angle $=0^{\circ}$ with the camera placed at a height of $1.5 \mathrm{~m}$ from the ground; the focal length was $8 \mathrm{~mm}$.

An illustrative result, displayed in Fig. 3, is the outperformance of the proposed Theil-Sen estimator as compared to linear regression based on the Pearson product-moment correlation coefficient. The green pixels in the right crop line are the annotated pixels obtained by considering the expected crop line as given by the application geometric transformations according to the intrinsic and extrinsic parameters and the margin of tolerance set to 150 pixels for each side around the expected crop line, making a total margin of 300 pixels. As we can easily infer, pixels far away from the central ones are weeds, i.e. they appear scattered in the inter-rows. Red line ${ }^{1}$ in the image is estimated by applying the Theil-Sen estimator and blue line is the one estimated by linear regression based on the Pearson product-moment correlation coefficient. As we can easily see, the best adjusting is achieved by the Theil-Sen's method. This is because it is robust enough against pixel dispersions. On the contrary, the regression-based method is sensitive to this kind of dispersion because it is based on the computation of minimum distances and the scattered pixels exert an important attractiveness.

The performance of the Theil-Sen estimator against the Pearson product-moment correlation coefficient is studied through a qualitative analysis, based on the human expert criterion because no ground truth images are available. By visual inspection of real crop row on the images, the expert determines the best adjustment of the estimated crop lines.

Because this approach is oriented to work in the future in realtime applications once the quality performance is achieved, we analyze the behavior of both estimators when applied to images with different resolutions, now under a quantitative analysis.

Fig. 4(a) and (b) represent an illustrative example representing an image of the 240 ones analyzed. The area of interest is delimited by landmarks with a wide of $2.25 \mathrm{~m}$ (covering three crop rows with inter-row spacing of $0.75 \mathrm{~m}$ ) and $4 \mathrm{~m}$ long. This area is the one to be processed during a normal operation of the agricultural robot.

The setting of the intrinsic and extrinsic parameters is the one described in Section 2.1. The camera system was placed $2 \mathrm{~m}$ from the area of interest, where a horizontal landmark line delimits the bottom part.

\footnotetext{
${ }^{1}$ For interpretation of color in the figures, the reader is referred to the web version of this article.
}

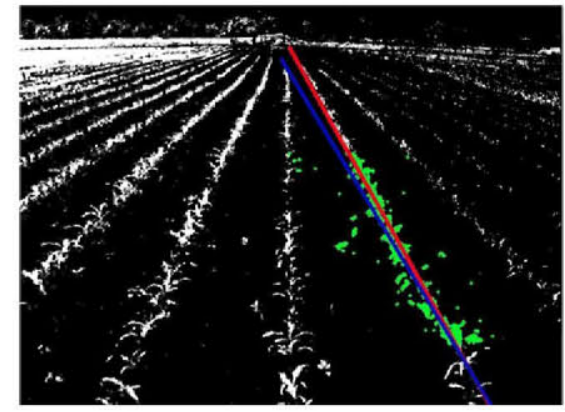

Fig. 3. Example of crop lines correction by Theil-Sen estimator (red line) and Pearson product-moment correlation coefficient (blue line) considering the dispersion of plants (green pixels).

\subsection{Qualitative analysis}

Fig. 4(a) displays in red those pixels representing green plants (crop and weeds). The expected crop lines, according to the system geometry based on extrinsic and intrinsic parameters, are drawn as yellow lines. A simple image inspection based on the human expert criterion allows us to infer that the expected crop lines do not match accurately with the real ones. This observation is particularly relevant in the left crop line. Perhaps this situation is due to the fact that this crop row was the outer line applied by the seeder machine and its location differs from the $0.75 \mathrm{~m}$ of the ideal interline spacing in maize fields. The deviation of the expected central and right crop lines from the real ones is less marked than in the left one. It is obvious that under this situation the expected crop lines need correction. The three real crop lines contain some gaps produced by errors during the sowing or perhaps because the maize seeds have not emerged.

Fig. 4(b) displays corrections of the yellow lines by applying both the Theil-Sen estimator (red lines) and regression through the Pearson product-moment correlation coefficient (blue lines). Based on the human expertise, it is easy to see how the three red lines are well adjusted by Theil-Sen. Indeed, they follow the central part of the furrow. Perhaps in the central crop line a slight deviation can be appreciated, which compared against the one produced by the Pearson product moment becomes irrelevant. The huge deviation produced by this last estimator in the central crop line is due to the presence of isolated patches (marked with the circle), probably weeds, which have been considered during the procedure of estimation. Something similar happens with respect the right crop lines, but the deviation it is less pronounced. This allows us to conclude that Theil-Sen works appropriately under this type of situations which are abundant in maize fields.

This is the general behavior observed in the set of 240 images analyzed, which allows us to verify the outperformance of the Theil-Sen estimator as compared to the Pearson product moment. The margin of tolerance used was again 150 for each side.

In order to detect the real crop lines over the image we have also applied the Hough transformation (Slaughter et al., 2008). Despite we apply geometric constraints considering the extrinsic and intrinsic parameters; the method produces abundant peaks on the accumulator cells, making difficult the selection of the correct one that identifies a crop row. A lot of lines, with different slopes, appear for a unique crop row. This requires a careful peak thresholding selection (Jones et al., 2009a, 2009b; Rovira-Más et al., 2005).

\subsection{Quantitative analysis}

From a point of view of quantitative analysis we compare the average percentage of success based on the human expert criterion 


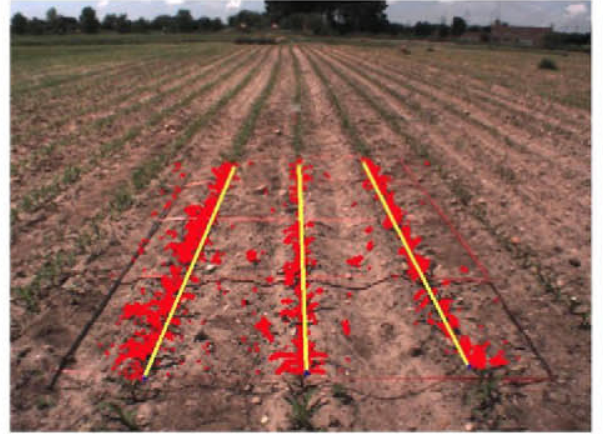

(a)

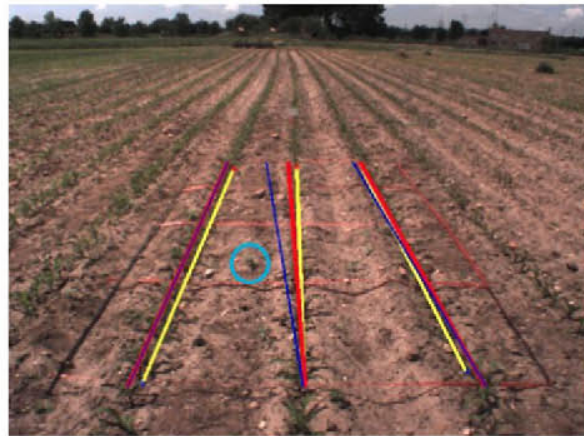

(b)

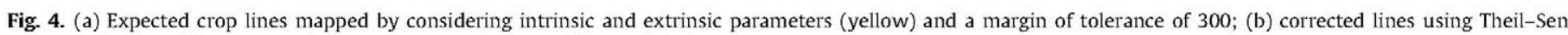
estimator (red) and Pearson product-moment correlation coefficient (blue).

Table 1

Averaged percentages of success and processing times (ms) for WA, Pearson and Theil-Sen for different size reductions and margins of tolerance.

\begin{tabular}{|c|c|c|c|c|c|c|c|c|c|c|}
\hline & \multirow{2}{*}{$\begin{array}{l}\text { Reduction } \\
\text { Margin of tolerance }\end{array}$} & \multicolumn{3}{|c|}{$0 \%$ (original image) } & \multicolumn{3}{|l|}{$67 \%$} & \multicolumn{3}{|l|}{$84 \%$} \\
\hline & & 50 & 100 & 150 & 50 & 100 & 150 & 50 & 100 & 150 \\
\hline \multirow[t]{3}{*}{ Percentage of success } & WA & 70 & 70 & 70 & 70 & 70 & 70 & 70 & 70 & 70 \\
\hline & Pearson & 78.2 & 83.1 & 72.9 & 80.2 & 83.1 & 79.3 & 81.1 & 82.3 & 69.3 \\
\hline & Theil-Sen & 86.1 & 93.1 & 94.1 & 85.2 & 91.3 & 84.6 & 83.8 & 83.1 & 77.8 \\
\hline \multirow[t]{3}{*}{ Time (ms) } & WA & 105 & 105 & 105 & 11.3 & 11.3 & 11.3 & 3.3 & 3.3 & 3.3 \\
\hline & Pearson & 9.3 & 9.5 & 9.9 & $8 \cdot 10^{-2}$ & $3.4 \cdot 10^{-1}$ & $7.5 \cdot 10^{-1}$ & $8.0 \cdot 10^{-4}$ & $2.0 \cdot 10^{-5}$ & $3.9 \cdot 10^{-5}$ \\
\hline & Theil-Sen & 5354 & 7506 & 9568 & 92 & 476 & 1440 & 30 & 98 & 230 \\
\hline
\end{tabular}

\section{GA + Otsu + THEIL-SEN Adjustment}

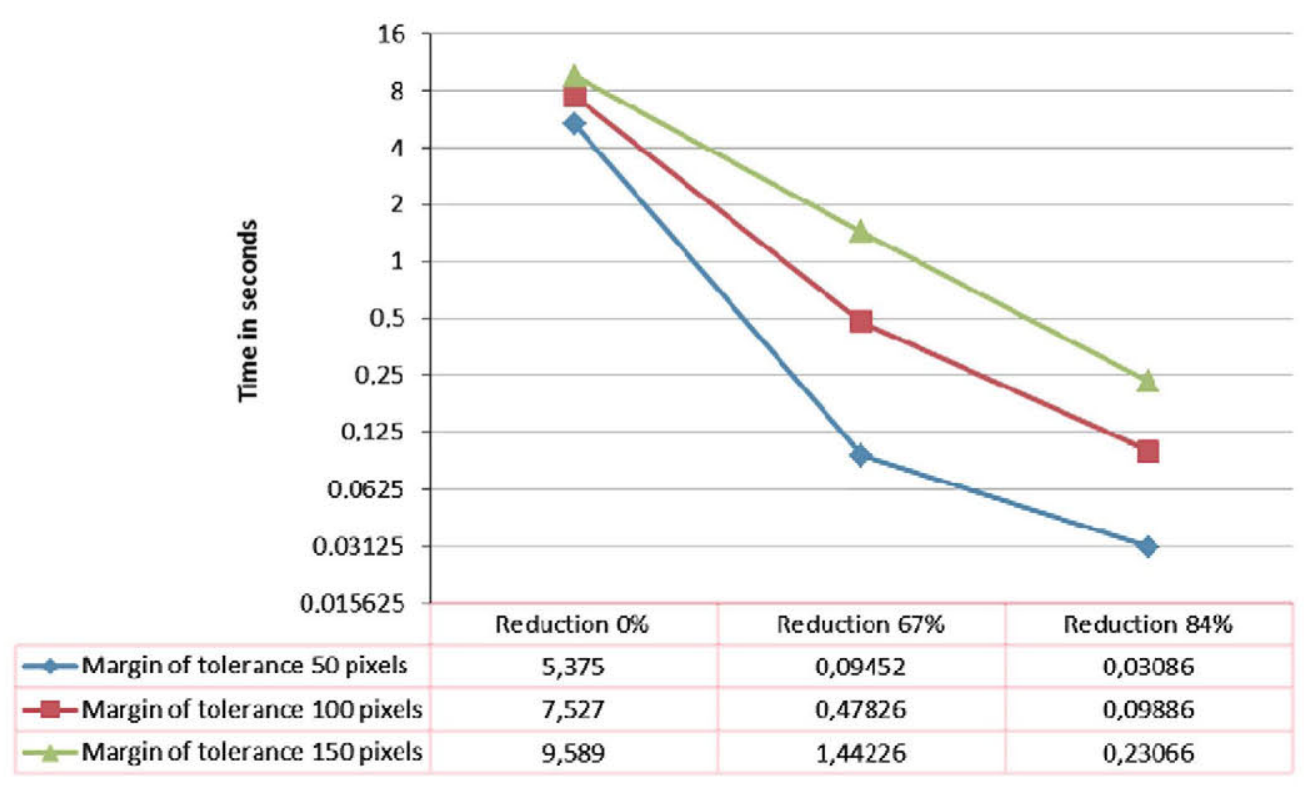

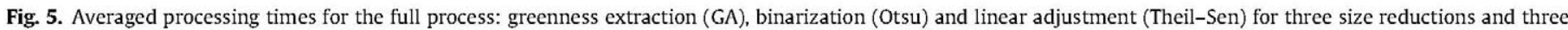
margins of tolerance.

for both Pearson and Theil-Sen and also without adjustment. Each image was visually analyzed by an expert to identify the accuracy between the adjust line and the real crop row considered as satisfactory by the expert. We also compute the computational cost measured in processing times for these three approaches. We call without adjustment (WA) the procedure involving ExG greenness extraction and Otsu based binarization, i.e. we only analyze the crop line detection obtained from a direct geometric mapping based on intrinsic and extrinsic parameters.
For Pearson and Theil-Sen, times displayed are exclusively the ones obtained for the specific adjustment, i.e. to obtain the total time they must be added to the one displayed without adjustment.

Because Theil-Sen is computational expensive but effective, we have reduced the resolution of the original image by down-sampling until to achieve values of $67 \%$ and $84 \%$ to verified the performances with and without reductions. Table 1 displays averaged percentages and computational times expressed in milliseconds for the set of original images available (i.e. with the $0 \%$ of 
reduction) and also for these images when their sizes are reduced until the $67 \%$ and $84 \%$ respectively, in horizontal and vertical sizes. For each reduction we display averaged percentages and times for three values (50,100 and 150) in what we call margin of tolerance. This margin represents the horizontal width used to search pixels representing crop and weeds around the expected crop line obtained by applying geometrical constraints (extrinsic and intrinsic parameters). Under this consideration all values for WA do not vary because here no search is required.

From Table 1 we can see that the best performance, in terms of accuracy, is achieved by the Theil-Sen approach as compared to WA and Pearson, because it obtains the best results. The best absolute performance of Theil-Sen, also in terms of accuracy, is obtained for the original images with a margin of 150 pixels. This is because these images contain the maximum information and this margin covers the necessary range to capture all information coming from crops being unaffected by weeds pixels. As the reduction increases the accuracy decreases, except for a reduction of $67 \%$ with margin of 100 pixels. The decreasing can be explained by the fact that the greater the reduction and tolerance, more pixels belonging to weeds are involved in the line estimation. The exception to this general behavior occurs for a reduction of $67 \%$ and a margin of 100 . We have tested different combinations of margins and reduction without apparent improvements with respect to the ones displayed.

Regarding times, it is obvious that the greater the image sizes and margins of tolerance the greater are processing times. This is because a greater number of pixels need to be processed. The main drawback for Theil-Sen is its high computational cost. Assuming the vision system captures an area of $4 \mathrm{~m}$ long the robot will need to navigate at speeds below of $4 / T(\mathrm{~m} / \mathrm{s})$ to gain time for image processing and actuation. $T$ represents the processing time. So, in the worst case $T=9.568 \mathrm{~s}$ and in the best case $T=0.476 \mathrm{~s}$. The corresponding speeds are $1.5 \mathrm{~km} / \mathrm{h}$ and $30.25 \mathrm{~km} / \mathrm{h}$, where the first one is acceptable for agricultural tasks and the second one is excessive. This means that it is unnecessary reductions of $67 \%$, i.e. reductions between 0 and 67 should be appropriate depending on the agricultural treatments.

For clarity, Fig. 5 displays averaged processing times, in seconds, over the set of images available for the full process including greenness extraction based on $E x G$, binarization through the Otsu's method and line adjustment based on the Theil-Sen estimator. As above, these values are obtained for the three values of reduction $(0 \%, 67 \%$ and $84 \%)$ and with the three margins of tolerance in pixels $(50,100$ and 150). As expected, processing times decrease as the reduction and margin of tolerance also decrease.

\section{Conclusions}

We propose a method for accuracy crop row detection in maize fields. The image is segmented to transform the original color image into a gray scale. Then a binarization process is carried out based on the Otsu's method. This allows identifying green plants which are white pixels in the binary image. According to the intrinsic and extrinsic parameters and applying perspective projection, we trace the expected crop lines over the original image. Considering that white pixels in the binary image are crop and weeds in the original one we follow the expected crop lines and explore in the horizontal direction to capture white pixels in the binary image. These pixels are the ones used for estimating a new crop line that can coincide with the expected one or not. If the new line does not match with the expected one, a correction is made, but if it matches the correct location is verified. The estimation is carried out by applying Theil-Sen and also a linear regression based on the Pearson product-moment correlation coefficient. We have verified Theil-Sen outperforms Pearson product-moment based on qualitative and quantitative analysis, in terms of accuracy, and it is acceptable from the point of view of the processing time.

Future improvements could be considered when high weed pressure is present in the image and a great number of weed patches invade the inter-row spacing.

\section{Acknowledgments}

The research leading to these results has been funded by the European Union's Seventh Framework Programme [FP7/20072013] under Grant Agreement no. 245986 in the Theme NMP-2009-3.4-1 (automation and robotics for sustainable crop and forestry management). The authors wish also to acknowledge the project the AGL2011-30442-C02-02, supported by the Ministerio de Economía y Competitividad of Spain within the Plan Nacional of $\mathrm{I}+\mathrm{D}+\mathrm{i}$.

\section{References}

Adichie, J. N. (1967). Estimates of regression parameters based on Rank tests. Annals of Mathematical Statistics, 38, 894-904.

Astrand, B. (2005). Vision based perception or mechatronic weed control. Doctor of Philosophy Thesis, Chalmers and Halmstad Universities, Sweden.

Astrand, B., \& Baerveldt, A. J. (2005). A vision based row-following system for agricultural field machinery. Mechatronics, 15, 251-269.

Billingsley, J., \& Schoenfisch, M. (1997). The succesful develepmnet of a vision guidance system for agriculture. Computers and Electronics in Agriculture, 16 $147-163$.

Bossu, J., Gêe, Ch., Guillemin, J. P., \& Truchetet, F. (2006). Development of methods based on double Hough transform and Gabor filtering to discriminate crop and weeds in agronomic images. In Proc. SPIE 18th annual symposium electronic imaging science and technology (Vol. 6070), paper no. 23, San Jose, USA.

Bossu, J., Gée, Ch., Jones, G., \& Truchetet, F. (2009). Wavelet transform to discriminate between crop and weed in perspective agronomic images. Computers and Electronics in Agriculture, 65, 133-143.

Burgos-Artizzu, X. P., Ribeiro, A., Tellaeche, A., Pajares, G., \& Fernández-Quintanilla, C. (2009). Improving weed pressure assessment using digital images from an experience-based reasoning approach. Computers and Electronics in Agriculture, $65,176-185$.

Davies, G., Casady, W., \& Massey, R. (1998). Precision agriculture: An introduction. Water Quality Focus Guide (WQ450, available on-line http:// extension.missouri.edu/explorepdf/envqual/wq0450.pdf).

Dytham, C. (2011). Choosing and using statistics: A biologist's guide (3rd ed.) 9781405198394. John Wiley and Sons (p. 230)

Fontaine, V., \& Crowe, T. G. (2006). Development of line-detection algorithms for local positioning in densely seeded crops. Canadian Biosystems Engineering, $48(7), 19-29$.

Fu, K. S., González, R. C., \& Lee, C. S. G. (1987). Robotics: Control, sensing, vision and intelligence. New York: McGraw-Hill.

Gée, Ch., Bossu, J., Jones, G., \& Truchetet, F. (2008). Crop/weed discrimination in perspective agronomic images. Computers and Electronics in Agriculture, 60, 49-59.

Guerrero, J. M., Pajares, G., Montalvo, M., Romeo, J., \& Guijarro, M. (2012). Support vector machines for crop/weeds identification in maize fields. Expert Systems with Applications, 39(12), 11149-11155. http://dx.doi.org/10.1016/ j.eswa.2012.03.040.

Guijarto, M., Pajares, G., Riomoros, I., Herrera, P. J., Burgos-Artizzu, X. P., \& Ribeiro, A. (2011). Automatic segmentation of relevant textures in agricultural images. Computers and Electronics in Agriculture, 75, 75-83.

Hague, T., Marchant, J. A., \& Tillett, D. (1997). A system for plant scale husbandry. Precision Agriculture, 635-642.

Hague, T., Tillet, N., \& Wheeler, H. (2006). Automated crop and weed monitoring in widely spaced cereals. Precision Agriculture, 1(1), 95-113.

Hampel, F. R., Rousseeuw, P. J., Ronchetti, E., \& Stahel, W. A. (1986). Robust statistics: The approach based on influence functions. New York: John Wiley.

Hartley, R., \& Zisserman, A. (2006). Multiple view geometry in computer vision. Oxford: Cambridge University Press.

Hough, P. V.C. (1962). A method and means for recognizing complex patterns. U.S Patent Office No. 3069654

Huber, P. J. (1981). Robust statistics. New York: John Wiley.

Ji, R., \& Qi, L. (2011). Crop-row detection algorithm based on random Hough transformation. Mathematical and Computer Modelling, 54, 1016-1020.

Jones, G., Gée, Ch., \& Truchetet, F. (2009a). Modelling agronomic images for weed detection and comparison of crop/weed discrimination algorithm performance. Precision Agriculture, 10, 1-15.

Jones, G., Gée, Ch., \& Truchetet, F. (2009b). Assessment of an inter-row weed infestation rate on simulated agronomic images. Computers and Electronics in Agriculture, 67, 43-50 
Kataoka, T., Kaneko, T., Okamoto, H., \& Hata, S. (2003). Crop growth estimation system using machine vision. In The 2003 IEEE/ASME internat. confference on advanced intelligent mechatronics.

Kendall, M. G. (1955). Rank correlation methods (2nd ed.). London: Charles Griffin and Company.

Kise, M., \& Zhang, Q. (2008). Development of a stereovision sensing system for 3D crop row structure mapping and tractor guidance. Biosystems Engineering, 101, 191-198.

Kise, M., Zhang, Q., \& Rovira-Más, F. (2005). A stereovision-based crop row detection method for tractor-automated guidance. Biosystems Engineering, 90(4), $357-367$.

Leemans, V., \& Destain, M. F. (2006). Application of the Hough transform for seed row location using machine vision. Biosystems Engineering, 94(3), 325-336.

López-Granados, F. (2011). Weed detection for site-specific weed management: mapping and real-time approaches. Weed Research, 51, 1-11.

Marchant, J. (1996). Tracking of row structure in three crops using image analysis. Computers and Electronics in Agriculture, 15, 161-179.

Massart, D. L., Vandeginste, B. G. M., Buydens, L. M. C., De Jong, S., Lewi, P. J., \& Smeyers-Verbeke, J. (1997). Single median method, In Handbook of chemometrics and qualimetrics: Part. Data handling in science and technology (vol. 20, 355-356), Elsevier.

Meyer, G. E., \& Camargo-Neto, J. (2008). Verification of color vegetation indices for automated crop imaging applications. Computers and Electronics in Agriculture, $63,282-293$.

Meyer, G. E., Hindman, T. W., \& Lakshmi, K. (1998). Machine vision detection parameters for plant species identification. Bellingham, WA: SPIE.

Montalvo, M., Pajares, G., Guerrero, J. M., Romeo, J., Guijarro, M., Ribeiro, A., et al (2012). Automatic detection of crop rows in maize fields with high weeds pressure. Expert Systems with Applications, 39(15), 11889-11897.

Mood, A. M. (1950). Introduction to the theory of statistics. New York: McGraw-Hill Book Company.

Neto, J. C. (2004). A combined statistical-soft computing approach for classification and mapping weed species in minimum tillage systems. Lincoln, NE: University of Nebraska.

Olsen, H. J. (1995). Determination of row position in small-grain crops by analysis of video images. Computers and Electronics in Agriculture, 12, 147-162.

Onyango, C. M., \& Marchant, J. A. (2003). Segmentation of row crop plants from weeds using colour and morphology. Computers and Electronics in Agriculture, 39, 141-155.

Otsu, N. (1979). A threshold selection method from gray-level histogram. IEEE Transactions on Systems Man and Cybernetics, 9, 62-66.

Pla, F., Sanchiz, J. M., Marchant, J. A., \& Brivot, R. (1997). Building perspective models to guide a row crop navigation vehicle. Image and Vision Computing, 15, 465-473.

Ribeiro, A., Fernândez-Quintanilla, C., Barroso, J., \& Garcia-Alegre, M. C. (2005). Development of an image analysis system for estimation of weed. In Proceedings of the 5th european conference on precision agriculture (5ECPA) (pp. 169-174).
Romeo, J., Pajares, G., Montalvo, M., Guerrero, J. M., Guijarro, M., \& Ribeiro, A. (2012). Crop row detection in maize fields inspired on the human visual perception. The Scientific World Joumal, 2012. http://dx.doi.org/10.1100/2012/484390. Article ID 484390,10 pages.

Rousseeuw, P. J. (1984). Least median of squares regression. Journal of American Statistical Association, 79, 871-880.

Rousseeuw, P. J., \& Leroy, A. M. (1987). Robust regression and outlier detection. New York: John Wiley.

Rovira-Más, F., Zhang, Q., \& Reid, J. F. (2008). Stereo vision three-dimensional terrain maps for precision agriculture. Computers and Electronics in Agriculture, 60 133-143.

Rovira-Más, F., Zhang, Q., Reid, J. F., \& Will, J D (2005). Hough-transform-based vision algorithm for crop row detection of an automated agricultural vehicle. Journal of Automobile Engineering Part D, 219, 999-1010.

Sainz-Costa, N., Ribeiro, A., Burgos-Artizzu, X., Guijarro, M., \& Pajares, G. (2011) Mapping wide row crops with video sequences acquired from a tractor moving at treatment speed. Sensors, 11, 7095-7109.

Sen, P. K. (1968). Estimates of the regression coefficient based on Kendall's Tau. Journal of American Statistical Association, 63, 1379-1389.

Sezgin, M. B., \& Sankur, B. (2004). Survey over image thresholding techniques and quantitative performance evaluation. Journal of Electronic Imaging, 13(1) 146-165.

Slaughter, D. C., Giles, D. K., \& Downey, D. (2008). Autonomous robotic weed control systems: A review. Computers and Electronics in Agriculture, 61, 63-78.

Søgaard, H. T., \& Olsen, H. J. (2003). Determination of crop rows by image analysis without segmentation. Computers and Electronics in Agriculture, 38, 141-158.

Stewart, C. V. (1999). Robust parameter estimation in computer vision. SIAM Review, 41(3), 513-537.

Tellaeche, A., Burgos-Artizzu, X. P., Pajares, G., \& Ribeiro, A. (2008). A vision-based method for weeds identification through the Bayesian decision theory. Pattern Recognition, 41, 521-530.

Tellaeche, A., Burgos-Artizzu, X., Pajares, G., Ribeiro, A., \& Fernăndez-Quintanilla, C. (2008). A new vision-based approach to differential spraying in precision agriculture. Computers and Electronics in Agriculture, 60(2), 144-155.

Tellaeche, A., Pajares, G., Burgos-Artizzu, X. P., \& Ribeiro, A. (2011). A computer vision approach for weeds identification through support vector machines. Applied Soft Computing, 11, 908-915.

Theil, H. (1950). A rank-invariant method of linear and polynomial regression analysis. In I, II, and III, Nederl. Akad. Wetensch. Proc., 53, 386-392, 521-525 and 1397-1412.

Vioix, J. B., Douzals, J. P., Truchetet, F., Assemat, L., \& Guillemin, J. P. (2002). Spatial and spectral method for weeds detection and localization. EURASIP JASP, 7 679-685.

Woebbecke, D. M., Meyer, G. E., von Bargen, K., \& Mortensen, D. A. (1995). Shape features for identifying young weeds using image analysis. Transactions on American Society of Agricultural Engineering, 38(1), 271-281. 